\title{
Fano resonances and Aharonov-Bohm effects in transport through a square quantum dot molecule
}

\author{
Z. Y. Zeng ${ }^{1,2}$, F. Claro ${ }^{1}$, and Alejandro Pérez ${ }^{1}$ \\ 1.Facultad de Física, Pontificia Universidad Católica de Chile, Casilla 306, Santiago 22, Chile \\ 2. Department of Physics, Hunan Normal University, Changsha 410081, China and \\ CCAST(World Laboratory). P. O. Box 8730, Beijing 100080, China
}

(October 25, 2018)

\begin{abstract}
We study the Aharonov-Bohm effect in a coupled $2 \times 2$ quantum dot array with two-terminals. A striking conductance dip arising from the Fano interference is found as the energy levels of the intermediate dots are mismatched, which is lifted in the presence of a magnetic flux. A novel five peak structure is observed in the conductance for large mismatch. The Aharonov-Bohm evolution of the linear conductance strongly depends on the configuration of dot levels and interdot and dot-lead coupling strengths. In addition, the magnetic flux and asymmetry between dot-lead couplings can induce the splitting and combination of the conductance peak(s).
\end{abstract}

PACS numbers: 73.23.-b,73.63.-b,73.63.Kv

Typeset using REVTEX 


\section{INTRODUCTION}

The famous effect predicted by Aharonov and Bohm (AB) in 1959 [1] concerns the essential influence of a vector potential on the interference pattern of two beams of electrons confined in multiply connected paths, over which the magnetic field is zero. It is manifested by the periodic oscillation of the conductance of a ring or a cylinder as a function of the enclosed magnetic flux $\Phi$ [2]. It has been shown that the AB effect also exists in some singly connected geometry such as a point contact or a disk shape in a two-dimensional electron gas, in which circulating edge states enclose a well-defined magnetic flux [3]. A significant hallmark in mesoscopic experiments is the phase measurements of the transmission amplitude through a quantum dot embedded in an AB ring in the Coulomb blockade regime 4 . It revived interest on the $\mathbf{A B}$ effect in condensed matter physics [5].

Quantum dots are highly tunable artificial mesoscopic structures [6], usually called artificial atoms or artificial molecules. Some novel physical properties, such as the Coulomb blockade and the turnstile effect, have been demonstrated in some elegant experiments [6]. At low temperatures, transport through a single quantum dot is dominated by resonant tunneling and Coulomb blockade. When the condition for resonant tunneling is not accessible, co-tunneling events involving the simultaneous tunneling of two or more electrons through a virtual intermediate level can be dominant [7]. When quantum dots are connected in series with tunneling barrier(s), it is expected that the coupling between dots plays a crucial role in the transport properties of the coupled structure. On the other hand, electron tunneling through the coupled dot system is very sensitive to the incoherent scattering, which has trivial effects on the transport properties of a single dot [8]. In the past decade, transport through coupled quantum dot systems has received considerable amount of attention [9 11].

In this paper, we investigate coherent electronic transport through four coupled quantum dots located at the four corners of a square enclosing a magnetic flux. This kind of coupled dot structure is the basic unit of two-dimensional quantum dot arrays. Our goal is to find out how the conductance of such a structure depends on the coupling strength between dots, 
the arrangement of dot levels and the magnetic flux. In the present work, we do not take into consideration the interdot and intradot electron-electron interactions. We also assume that just one level is relevant on each dot. This allows us, on the one hand, to obtain some exact analytical results. On the other hand, we can gain much more clear physical insight into the dependence of the conductance of the system on the structure parameters (interdot couplings and dot levels) and the magnetic flux. Though the model we consider is simple, some novel and interesting results are obtained arising from the interplay of the specific configuration of dot levels and interdot couplings, and the magnetic flux.

The rest of the paper is organized as follows. In Sec. II we derive the current through the $2 \times 2$ quantum dot array using the Keldysh nonequilibrium Green's function formalism and a recursive Green's function technique. Section III presents the linear conductance spectra of the dot array in the symmetric and asymmetric coupling case. We also investigate in detail the Ahoronov-Bohm oscillation of the linear conductance in some specific configurations of the dot levels and the interdot couplings. Our concluding remarks are given in Sec. IV.

\section{FORMULATION}

We consider four quantum dots in a square array [12 enclosing a magnetic flux $\Phi$ (Fig. 1), with two dots facing each other connected to the left and right leads. For simplicity, we ignore the intra- and inter-dot Coulomb interactions, and assume that just one energy level is relevant at each dot. Then the hamiltonian describing such a system is

$$
\begin{aligned}
H & =H_{\text {Dot }}+H_{\text {Lead }}+H_{\text {Dot-Lead }}, \\
H_{\text {Dot }} & =\sum_{i=1}^{4} \epsilon_{i} d_{i}^{+} d_{i}+\left(t_{12} d_{1}^{+} d_{2}+t_{13} e^{i \phi} d_{1}^{+} d_{3}+t_{24} d_{4}^{+} d_{2}+t_{34} d_{4}^{+} d_{3}+\text { h.c. }\right), \\
H_{\text {Lead }} & =\sum_{k \in L} \epsilon_{k} a_{k}^{+} a_{k}+\sum_{p \in R} \epsilon_{p} b_{p}^{+} b_{p}, \\
H_{\text {Dot-Lead }} & =\sum_{k \in L}\left(V_{k 1} a_{k}^{+} d_{1}+V_{k 1}^{*} d_{1}^{+} a_{k}\right)+\sum_{p \in R}\left(W_{p 4} b_{p}^{+} d_{4}+W_{p 4}^{*} d_{4}^{+} b_{p}\right),
\end{aligned}
$$

where $\epsilon_{i}$ is the energy level of $\operatorname{dot} i, a_{k}\left(a_{k}^{+}\right), b_{p}\left(b_{p}^{+}\right)$and $d_{i}\left(d_{i}^{+}\right)(i=1,2,3,4)$ are the annihilation (creation) operators of electrons in the left(L) lead, $\operatorname{right}(\mathrm{R})$ lead and quantum dot $i$, 
and $t_{i j}$ and $V_{k 1}\left(W_{p 4}\right)$ are the inter-dot and dot-lead coupling matrix elements, respectively. In Eq. (1), a factor $e^{i \phi}\left(\phi=2 \pi \Phi / \Phi_{0}, \Phi_{0}=h / e\right)$ is attached to $t_{13}$ to account for the magnetic flux $\Phi$ through the structure.

The current flowing from the left lead to the structure is 13

$$
J_{L}=\frac{2 i e}{\hbar} \int \frac{d \epsilon}{2 \pi} \Gamma_{L}(\epsilon)\left\{f_{L}(\epsilon)\left[G_{d_{1} d_{1}}^{r}(\epsilon)-G_{d_{1} d_{1}}^{a}(\epsilon)\right]+G_{d_{1} d_{1}}^{<}(\epsilon)\right\}
$$

The notation we use throughout is $G_{X Y}^{r, a}\left(t, t^{\prime}\right)=\mp i \theta\left( \pm t \mp t^{\prime}\right)<\left\{X(t), Y^{+}\left(t^{\prime}\right)\right\}>$, $G_{X Y}^{<}\left(t, t^{\prime}\right)=i<Y^{+}\left(t^{\prime}\right) X(t)>, X, Y=a_{k}, b_{P}, d_{i}(i=1,2,3,4), \Gamma_{L}(\epsilon)=2 \pi\left|V_{k 1}\right|^{2} \delta\left(\epsilon-\epsilon_{k}\right)$, while $f_{L}(\epsilon)=\left[1+e^{\left(\epsilon-\mu_{L}\right) / k_{B} T}\right]^{-1}$ is the Fermi-Dirac distribution function of the left lead, with chemical potential $\mu_{L}$. The factor of 2 in Eq. (2) is due to the spin degeneracy.

Following the recursive decoupling technique developed in Ref. [14], the retarded Green's function appearing in Eq. (2) $G_{d_{1} d_{1}}^{r}(\epsilon)$ can be calculated from Dyson's equation

$$
\begin{aligned}
G_{d_{1} d_{1}}^{r} & =\left[\epsilon-\epsilon_{1}-\Sigma_{d_{1}}^{r}\right]^{-1} \\
\Sigma_{d_{1}}^{r} & =-\frac{i}{2} \Gamma_{L}+\left|t_{12}\right|^{2} \tilde{G}_{d_{2} d_{2}}^{r}+\left|t_{13}\right|^{2} \tilde{G}_{d_{3} d_{3}}^{r}+t_{12} t_{13}^{*} e^{-i \phi} \tilde{G}_{d_{2} d_{3}}^{r}+t_{12}^{*} t_{13} e^{i \phi} \tilde{G}_{d_{3} d_{2}}^{r} .
\end{aligned}
$$

Here and in what follows $\tilde{G}_{d_{i} d_{j}}^{r, a<}$ denote the Green's function which is decoupled from the quantum dot $k(k=\min (i, j)-1)$. In the above and following equations we drop the argument $\epsilon$, and recover it wherever necessary. Following a similar recursive decoupling method, one has

$$
\begin{aligned}
\tilde{G}_{d_{2} d_{2}}^{r} & =\frac{\left(\epsilon-\epsilon_{3}\right)\left(\epsilon-\epsilon_{4}+\frac{i}{2} \Gamma_{R}\right)-t_{34} t_{34}^{*}}{\left(\epsilon-\epsilon_{2}\right)\left(\epsilon-\epsilon_{3}\right)\left(\epsilon-\epsilon_{4}+\frac{i}{2} \Gamma_{R}\right)-t_{24} t_{24}^{*}\left(\epsilon-\epsilon_{3}\right)-t_{34} t_{34}^{*}\left(\epsilon-\epsilon_{2}\right)}, \\
\tilde{G}_{d_{3} d_{3}}^{r} & =\frac{\left(\epsilon-\epsilon_{2}\right)\left(\epsilon-\epsilon_{4}+\frac{i}{2} \Gamma_{R}\right)-t_{24} t_{24}^{*}}{\left(\epsilon-\epsilon_{2}\right)\left(\epsilon-\epsilon_{3}\right)\left(\epsilon-\epsilon_{4}+\frac{i}{2} \Gamma_{R}\right)-t_{24} t_{24}^{*}\left(\epsilon-\epsilon_{3}\right)-t_{34} t_{34}^{*}\left(\epsilon-\epsilon_{2}\right)}, \\
\tilde{G}_{d_{2} d_{3}}^{r} & =\frac{t_{24} t_{34}^{*}}{\left(\epsilon-\epsilon_{2}\right)\left(\epsilon-\epsilon_{3}\right)\left(\epsilon-\epsilon_{4}+\frac{i}{2} \Gamma_{R}\right)-t_{24} t_{24}^{*}\left(\epsilon-\epsilon_{3}\right)-t_{34} t_{34}^{*}\left(\epsilon-\epsilon_{2}\right)}, \\
\tilde{G}_{d_{3} d_{2}}^{r} & =\frac{t_{34} t_{24}^{*}}{\left(\epsilon-\epsilon_{2}\right)\left(\epsilon-\epsilon_{3}\right)\left(\epsilon-\epsilon_{4}+\frac{i}{2} \Gamma_{R}\right)-t_{24} t_{24}^{*}\left(\epsilon-\epsilon_{3}\right)-t_{34} t_{34}^{*}\left(\epsilon-\epsilon_{2}\right)}
\end{aligned}
$$

where $\Gamma_{R}(\epsilon)=2 \pi\left|W_{p 4}\right|^{2} \delta\left(\epsilon-\epsilon_{p}\right)$. In the following we will assume that the coupling matrix elements $\left(V_{k 1}, W_{p 4}\right.$ and $\left.t_{i j}\right)$ are real since the tunnel rates just depend on the amplitude of the coupling matrix elements. Substitution of Eqs. (5)-(8) into Eq. (4) yields 
$\Sigma_{d_{1}}^{r}(\epsilon)=-\frac{i}{2} \Gamma_{L}+\frac{\left(\epsilon-\epsilon_{4}+\frac{i}{2} \Gamma_{R}\right)\left[t_{12}^{2}\left(\epsilon-\epsilon_{3}\right)+t_{13}^{2}\left(\epsilon-\epsilon_{2}\right)\right]-\left(t_{12}^{2} t_{34}^{2}+t_{13}^{2} t_{24}^{2}\right)+2 \cos \phi t_{12} t_{13} t_{24} t_{34}}{\left(\epsilon-\epsilon_{2}\right)\left(\epsilon-\epsilon_{3}\right)\left(\epsilon-\epsilon_{4}+\frac{i}{2} \Gamma_{R}\right)-t_{24}^{2}\left(\epsilon-\epsilon_{3}\right)-t_{34}^{2}\left(\epsilon-\epsilon_{2}\right)}$.

It can be seen from the above expression that the $\mathbf{A B}$ effect manifests itself in an additional term $2(\cos \phi-1) t_{12} t_{13} t_{24} t_{34}$ in the self-energy $\Sigma_{d_{1}}$ of dot 1 Green's function, the dominant contribution to the spectral density of the structure.

We now have to calculate the lesser Green's function $G_{d_{1} d_{1}}^{<}$. It can be obtained from the Keldysh formula

$$
G_{d_{1} d_{1}}^{<}=G_{d_{1} d_{1}}^{r} \Sigma_{d_{1}}^{<} G_{d_{1} d_{1}}^{a}=\Sigma_{d_{1}}^{<}\left|G_{d_{1} d_{1}}^{r}\right|^{2}
$$

where

$$
\Sigma_{d_{1}}^{<}=i f_{L}(\epsilon) \Gamma_{L}+\sum_{i=2,3} t_{1 i}^{2} \tilde{G}_{d_{i} d_{i}}^{<}+t_{12} t_{13} e^{-i \phi} \tilde{G}_{d_{2} d_{3}}^{<}+t_{12} t_{13} e^{i \phi} \tilde{G}_{d_{3} d_{2}}^{<}
$$

The lesser Green's functions $\tilde{G}_{d_{i} d_{j}}^{<}(i, j=2,3)$ can be calculated from the following Keldysh equation

$$
\tilde{G}_{d_{i} d_{j}}^{<}=\sum_{m, n=2,3} \tilde{G}_{d_{i} d_{m}}^{r} \tilde{\Sigma}_{d_{m} d_{n}}^{<} \tilde{G}_{d_{n} d_{j}}^{r}, \quad i, j=2,3
$$

where

$$
\tilde{\Sigma}_{d_{m} d_{n}}^{<}=\frac{i f_{R}(\epsilon) \Gamma_{R} t_{m 4} t_{n 4}}{\left(\epsilon-\epsilon_{4}\right)^{2}+\Gamma_{R}^{2} / 4} . \quad m, n=2,3
$$

Here $f_{R}(\epsilon)=\left[1+e^{\left(\epsilon-\mu_{R}\right) / k_{B} T}\right]^{-1}$ is the Fermi-Dirac distribution function of the right lead, with chemical potential $\mu_{R}$. It is worth noticing that the above procedure of calculating the various kinds of Green's functions can be verified to give the same results as the equationof-motion method [15]. Combining Equations (6) - (10) we find

$$
G_{d_{1} d_{1}}^{<}=\left(i f_{L}(\epsilon) \Gamma_{L}-2 i f_{R}(\epsilon) \operatorname{Im}\left[\Sigma_{d_{1}}^{r}+\frac{i}{2} \Gamma_{L}\right]\right)\left|G_{d_{1} d_{1}}^{r}\right|^{2} .
$$

Notice that

$$
G_{d_{1} d_{1}}^{r}-G_{d_{1} d_{1}}^{a}=\left(\frac{1}{G_{d_{1} d_{1}}^{a}}-\frac{1}{G_{d_{1} d_{1}}^{r}}\right)\left|G_{d_{1} d_{1}}^{r}\right|^{2}=2 i \operatorname{Im}\left[\Sigma_{d_{1}}^{r}\right]\left|G_{d_{1} d_{1}}^{r}\right|^{2}
$$


Substituting Eqs. (14) and (15) into Eq. (2), one then obtains the current entering the structure from the left lead,

$$
J=J_{L}=\frac{2 e}{h} \int d \epsilon\left[f_{L}(\epsilon)-f_{R}(\epsilon)\right] \mathcal{T}(\epsilon)
$$

in which

$$
\begin{aligned}
\mathcal{T}(\epsilon) & =\frac{\Gamma_{L}(\epsilon) \Gamma_{R}(\epsilon)}{\Gamma_{L}(\epsilon)+\Gamma_{R}(\epsilon)} \rho(\epsilon), \\
\rho(\epsilon) & =-\frac{2\left(\Gamma_{L}(\epsilon)+\Gamma_{R}(\epsilon)\right)}{\Gamma_{R}(\epsilon)} \operatorname{Im}\left[\Sigma_{d_{1}}^{r}(\epsilon)+\frac{i}{2} \Gamma_{L}(\epsilon)\right]\left|\frac{1}{\epsilon-\epsilon_{1}-\Sigma_{d_{1}}^{r}(\epsilon)}\right|^{2}
\end{aligned}
$$

is the transmission probability for an electron passing through the quantum dot square structure. Equations (16) and (17) are the central results of this work. It can be seen that the transmission probability and current are proportional to the dot-lead couplings $\left(\Gamma_{L}, \Gamma_{R}\right)$ and a generalized spectral density function $\rho(\epsilon)$ of the system. Note that the current includes contributions from the upper arm path $(1 \rightarrow 2 \rightarrow 4)$, and the bottom arm path $((1 \rightarrow 3 \rightarrow 4)$, which interfere with each other. At zero temperature, the conductance in the linear regime limit $\mu_{L} \approx \mu_{R} \rightarrow \epsilon_{F}$ becomes

$$
\mathcal{G}(\Phi)=\frac{2 e^{2}}{h} \frac{\Gamma_{L} \Gamma_{R}}{\Gamma_{L}+\Gamma_{R}} \rho\left(\epsilon_{F}\right)
$$

In obtaining Eq.(18) the wide-bandwidth approximation 13 has been used, i.e., the linewidths $\Gamma_{L}$ and $\Gamma_{R}$ are constants independent of energy. From the expression of the self-energy (9) one can find that the linear conductance $\mathcal{G}(\Phi)$ is a periodic function of the magnetic flux $\Phi$, with periodicity $\Phi_{0}=h / e$, and $\mathcal{G}(\Phi)=\mathcal{G}(-\Phi)$. These observations are consistence with the general results of the two-terminal setup of the $\mathbf{A B}$ ring [5].

When quantum dot 2 ( or 3 ) is decoupled from the system, i.e., $t_{12}=t_{24}=0$ ( or $t_{13}=t_{34}=0$ ), we recover the results for the coupled triple quantum dot chain [14]

$$
J=J_{L}=\frac{2 e}{h} \int d \epsilon\left[f_{L}(\epsilon)-f_{R}(\epsilon)\right] \mathcal{T}_{3}(\epsilon)
$$

where the transmission probability is

$$
\mathcal{T}_{3}(\epsilon)=\Gamma_{L} \Gamma_{R}\left|t_{1 i}\right|^{2}\left|t_{i 4}\right|^{2} \mathcal{A}(\epsilon) . \quad i=2 \quad(\text { or } 3)
$$


Here

$$
\begin{aligned}
\mathcal{A}(\epsilon) & =\frac{\left[\left(\epsilon-\epsilon_{4}\right)^{2}+\Gamma_{R}^{2} / 4\right] /\left\{\left[\left(\epsilon-\epsilon_{i}\right)\left[\left(\epsilon-\epsilon_{4}\right)^{2}+\Gamma_{R}^{2} / 4\right]-t_{i 4}^{2}\left(\epsilon-\epsilon_{4}\right)\right]^{2}+\left|t_{R}\right|^{4} \Gamma_{R}^{2} / 4\right\}}{\left(\epsilon-\epsilon_{1}-t_{1 i}^{2} \operatorname{Re} \mathcal{B}(\epsilon)^{2}+\left(\Gamma_{L}-2 t_{1 i}^{2} \operatorname{Im\mathcal {B}}(\epsilon)^{2} / 4\right.\right.}, \\
i & =2(\text { or 3) }
\end{aligned}
$$

where

$$
\mathcal{B}(\epsilon)=\left(\epsilon-\epsilon_{i}-\frac{t_{i 4}^{2}}{\epsilon-\epsilon_{4}+\frac{i}{2} \Gamma_{R}}\right)^{-1} . \quad i=2 \quad(\text { or } 3)
$$

When the levels of the three dots are aligned, we find the condition for complete transmission $\left(\mathcal{T}_{3}=1\right)$ to be

$$
\frac{t_{1 i}^{2}}{t_{i 4}^{2}}=\frac{\Gamma_{L}}{\Gamma_{R}} . \quad i=2 \quad(\text { or } 3)
$$

It can be expected that the criteria for complete resonant transmission or maximum peak conductance plays an important role in the formation of conductance peaks with the conductance quanta $2 e^{2} / h$ for the square quantum dot array.

\section{LINEAR CONDUCTANCE CHARACTERISTICS}

\section{A. Symmetric coupling between dots and leads}

It is well known that the two-terminal conductance of an $\mathbf{A B}$ ring is a periodic even function of the enclosed magnetic flux $\Phi$ [5]. The oscillation of the conductance, with periodicity $h / e$, results from the quantum interference between the paths located in the upper and lower arms of the ring. For electron tunneling through a single dot, the conductance

peak reaches its maximum when $\Gamma_{L}=\Gamma_{R}$. In the coupled double quantum dot structure with aligned energy levels, the conductance peak reach a maximum value and splits into a double peak with peak separation $2\left(t^{2}-\Gamma^{2} / 4\right)^{1 / 2}$ when the interdot coupling $t$ becomes larger than the width of the peak $\Gamma$ in the symmetric dot-lead coupling case [14]. For the three coupled dot string, the situation is more complicated, though the condition for maximum conductance peak is the simple Eq. (23) since the levels of the dots are aligned. We expect 
that the conductance peak will split into three maxima when the interdot coupling becomes larger than the width of the peak, as in the double dot case.

For further comparison, we first calculate the linear conductance of the quantum dot array in the absence of the magnetic flux $\Phi$ and in the symmetric dot-lead coupling case $\left(\Gamma_{L}=\Gamma_{R}\right)$. Throughout this paper, energy and coupling strengths are measured in units of $\Gamma_{L}=\Gamma$. The results for aligned levels and various kinds of interdot coupling configurations are shown in Fig. 2 (a), while Fig. 2 (b,c) are for different arrangements of the dot levels. When the four dot levels are aligned, three conductance peaks appear if $t_{12}=t_{13}$ and $t_{24}=t_{34}$. Then the conductance possesses the same three resonant peak structure as the three dot string. One can also find that the criteria for complete resonant transmission for the three-dot systems manifests itself in our four dot structure. This is not strange since the conductance in our case is simply the superposition of the contributions from the upper and lower three-dot arms. When $t_{12}=t_{34}<t_{13}=t_{24}$, the situation becomes different. In this case, one can consider the dot structure as a system of two coupled quantum dot dimers. One observes in the conductance spectra four peaks, arranged in two groups separated by a conductance gap. The separation between the two group centers is mainly determined by the larger interdot coupling, and the peak separation of each group by the smaller one. The peak and group center separations have trivial dependence on the dot-lead couplings $\Gamma_{L}$ and $\Gamma_{R}$ in the coupled quantum dimers case. The conductance gap is introduced by the larger coupling strength between the two dimers and can not be lifted by a magnetic flux, contrary to the case of the Fano conductance dip discussed below. The gap can be broadened by increasing the coupling strength(s) $t_{12}\left(\right.$ and/or $\left.t_{24}\right)$ between the two dimers.

Figures 2 (b,c), displaying cases of mismatched levels, show more interesting conductance spectra. When $t_{12}=t_{13}=t_{24}=t_{34}$ a striking novel conductance dip $(\mathcal{G}=0)$ appears halfway between the energy levels of dots 2 and 3 (Fig. 2(c) and dashed line in 2(b)) if these levels are different. When $t_{12}=t_{24}<<t_{13}=t_{34}$ the conductance dip is pinned at the energy level of the quantum dot with small couplings to the system (solid line in Fig. 2 (b)). The middle peak appears split into two asymmetric peaks. The asymmetry 
between the two split peaks depends on the mismatch between the energy levels of dots 2 and 3, as well as the arrangement of the levels of other dots 1 and 4 . Novel five spikes ( the dashed line in Fig. $2(\mathrm{~b})$ ) in the conductance can be seen if the mismatch between the levels of dot 2 and 3 is large enough. The dip results from a Fano interference [16] between two distinct current paths, one being direct nonresonant and the other nondirect resonant 17, 18]. The conductance can be generally written 17 as $\mathcal{G}=\mathcal{G}_{\text {non }} \frac{(\tilde{\epsilon}+q)^{2}}{\tilde{\epsilon}^{2}+1}$, where $\mathcal{G}_{\text {non }}$ is the nonresonant conductance, $\tilde{\epsilon}=\epsilon-\epsilon_{\text {reso }}$, and $q$ is the 'Fano parameter' assessing the asymmetry of the lineshape. Two limiting cases exist [Ref. 17(b)]: (a) $q \rightarrow \infty$, with a dominating resonant transmission resulting in Breit-Wigner resonances and, (b) $q \rightarrow 0$, with a dominating nonresonant transmission leading to asymmetric dips. Since here $t_{12}=$ $t_{13}=t_{24}=t_{34}$, one can consider either the arm consisting of dot 2 or 3 as the resonant (nonresonant) path. This special symmetry locates the Fano-type conductance dip at neither the level of dot 2 nor that of dot 3, but halfway between them. On the other hand, when $t_{12}=t_{13}=t_{24}=t_{34}$, we find from Eqs. (9), (17) and (18) that $\mathcal{G} \propto \frac{2 \epsilon_{F}-\epsilon_{2}-\epsilon_{3}}{\left(\epsilon_{F}-\epsilon_{2}\right)\left(\epsilon_{F}-\epsilon_{3}\right)}$. It is evident that two resonance peaks exist when $\epsilon_{F}=\epsilon_{2}$ and $\epsilon_{F}=\epsilon_{3}$, while $\mathcal{G}=0$ when $\epsilon_{F}=\left(\epsilon_{2}+\epsilon_{3}\right) / 2$, which is just the position of the Fano conductance dip. For large mismatch between energy levels of dots 2 and 3, Fano interference between the two paths develops both a conductance dip and a peak halfway between these levels $\epsilon_{2}$ and $\epsilon_{3}$. One can then observe a five peak structure in the conductance. When the levels of dots 2 and 3 are matched, the resonant peak structure of the dot systems behaves to a large extent the same way as the coupled three-dot system [14].

Now we turn to study the effect of the magnetic flux $\Phi$ on the conductance spectra of our square dot system. Figure 3 exhibits clearly the AB oscillations of the conductance as a function of the magnetic flux $\phi$ within a period at $\epsilon_{F}=0.1 \Gamma$. When the two arms of the dot ring are symmetric, i.e., the dot levels and interdot couplings are arranged to be the same, the conductance $\mathcal{G}$ vanishes at $\phi=\pi$ (Fig. 3(a) and the solid line in Fig. $3(\mathrm{c}))$. In the case of asymmetric arms, the conductance is nonzero within the whole period. The vanishing of $\mathcal{G}$ at $\phi=\pi$ results from the complete destructive interference between 
the two symmetric arms, which will be analyzed in more detail below. If the two arms are not symmetric, complete destructive interference is never achievable, and the conductance is always nonzero. The more asymmetry between the two arms, the larger the conductance near $\phi=\pi$. To get a clearer insight into the influence of a magnetic flux on $\mathcal{G}$, we give in Figs. 4 and 5 the conductance of the structure as a function of the Fermi energy for different values of magnetic flux within half a period $(\phi=0, \pi / 4,3 \pi / 4, \pi)$, and different configurations of interdot couplings and dot levels. The dependence of the conductance on the magnetic flux $\Phi$ is apparent. The magnitude of the $\mathbf{A B}$ oscillation strongly depends on the matching condition of the dot levels and the configuration of the interdot couplings. It also differs for different pinning positions of the Fermi energy at the leads. The presence of a magnetic flux can lead to the splitting (Fig. 5(b)) and combination (Fig. 4(c) and Figs. $5(\mathrm{a}, \mathrm{c}, \mathrm{d}))$ of conductance peak $(\mathrm{s})$ since it changes the interference between the two current paths, as determined by term $2(\cos \phi-1) t_{12} t_{13} t_{24} t_{34}$ in Eq. (9). Another striking effect induced by the magnetic flux is that the central conductance peak, if present (Fig. 4), becomes a conductance dip when the levels of dots 2 and 3 are aligned, while the Fanotype dip appearing in the case of mismatched levels is lifted (Fig. 5), contrary to the usual conductance gap which survives in the presence of a magnetic flux (Fig. 4 (c)). This feature is typically a result of the modified Fano phase interference by the magnetic flux. When $\phi=\pi$, the conductance is depressed everywhere if $\epsilon_{2}=\epsilon_{3}$ and $t_{12} t_{24}=t_{13} t_{34}$, as shown in Fig. 4 and Fig. 5 (b). The overall depression of the conductance results from the complete destructive interference between the upper-arm and lower-arm current paths. When $\epsilon_{2}=\epsilon_{3}=\epsilon$ and $t_{12} t_{24}=t_{13} t_{34}$, one finds $\mathcal{G} \propto 2(1+\cos \phi) t_{12}^{2} t_{24}^{2}$, which implies zero conductance for any value of the Fermi energy if $\phi=\pi$. We can also analyze it from the interference pattern of the two current paths containing either dot 2 or 3 . The respective transmission amplitudes $T_{2}=t_{12} t_{24} /\left(\epsilon_{F}-\epsilon_{2}+i 0^{+}\right)$and $T_{3}=t_{13} t_{34} e^{i \phi} /\left(\epsilon_{F}-\epsilon_{3}+i 0^{+}\right)$ equal $T /\left(\epsilon_{F}-\epsilon+i 0^{+}\right)$and $T e^{i \phi} /\left(\epsilon_{F}-\epsilon+i 0^{+}\right)$, respectively, when $t_{12} t_{24}=t_{13} t_{34}=T$ and $\epsilon_{2}=\epsilon_{3}=\epsilon$. Then the total transmission probability $\mathcal{T}=\left|T_{2}+T_{3}\right|^{2}$ for electron tunneling through the dot structure is proportional to $2 T^{2}(1+\cos \phi) \delta\left(\epsilon_{F}-\epsilon\right)$, which is zero when 
$\phi=\pi$.

\section{B. Asymmetric coupling between dots and leads}

In the preceding subsection we have reported in detail the linear conductance spectra in the absence and presence of a magnetic flux, in the limit of symmetric dot-lead couplings. There we saw symmetrically-located conductance peaks if the dot levels are aligned, which is a consequence of the particle-hole symmetry. In this subsection, we want to study how the conductance is modified if the dot-lead couplings are asymmetric.

We model the asymmetry between the dot-lead coupling $\Gamma_{L}$ and $\Gamma_{R}$ by setting $\Gamma_{R}=\Gamma_{L} / 2$. Figures 6 and 7 present the comparison of conductance spectra in the cases of symmetric (thin lines) and asymmetric (thick lines) dot-lead couplings, with structure parameters the same as in Fig. 4 and 5, respectively. A surprising feature in these figures is that the asymmetry between dot-lead couplings can remove the symmetry of the conductance spectra around some energy when the energy levels of dots are not aligned (Figs. 7 (c,d)), while it can not if they are aligned (Fig. 6). Also depending on the value of the magnetic flux $\phi$, the conductance in the case of asymmetric dot-lead couplings is lifted (Figs. 6 (a,c), $\phi=3 \pi / 4$ ) or suppressed (Figs. 6 (a,c), $\phi=\pi / 4$ and Fig. 7 (a) $\phi=\pi / 4,3 \pi / 4$ ), as compared to the symmetric case. We also observe another interesting phenomenon. The conductance changes in a nonuniform way when the dot-lead couplings become asymmetric. This is reflected in the observation that some conductance peaks are lifted while the others are suppressed (Fig. 6 (b) and Figs. 7 (c,d)). In addition, we find that the asymmetric dot-lead couplings would introduce a splitting of the conductance peak (Fig. 6 (b), Figs. 7 (c,d)). It is worth mentioning that the position of the conductance peaks remain nearly unshifted when the dot-lead couplings become asymmetric.

These features can be understood for linear conductance from the analysis of the generalized spectral function $\rho(\epsilon)$ in Equation (17), which depends on the dot-lead couplings $\Gamma_{L}$ and $\Gamma_{R}$. In the case of a single quantum dot,a difference in the value of the dot-lead cou- 
plings $\Gamma_{L}$ and $\Gamma_{R}$ does not change the position of the resonant transmission or conductance peak, but does alter its height. In our case, the dependence on $\Gamma_{L}$ and $\Gamma_{R}$ of the spectral function or conductance is much more complicated. However, it appears that a difference between $\Gamma_{L}$ and $\Gamma_{R}$ does also modify the weight of the peaks in the generalized spectral function $\rho(\epsilon)$ in a complicated way dependent on the applied magnetic flux. The position of the conductance peaks is kept intact by the difference between $\Gamma_{L}$ and $\Gamma_{R}$.

\section{CONCLUSIONS}

To sum up, we have investigated the transport properties in the linear regime, of a two-terminal quantum dot square enclosing a magnetic flux. Although our results are not directly applicable to more complex arrays involving more conducting paths and dots, we believe that our study will provide insight into the physics of interference that such arrays will exhibit. Our main results may be summarized as follows: (1) The AB oscillations of the conductance strongly depends on the configurations of dot levels and interdot and dot-lead couplings, along with the position of the Fermi energy of the leads; (2) a striking conductance dip is developed due to the Fano interference when the levels of the intermediate dots are mismatched, and a novel five peak structure is observed for large mismatch; (3) the magnetic flux and an asymmetric dot-lead coupling can induce the splitting and combination of the conductance peak(s); (4) the interplay of a magnetic flux and asymmetry between dot-lead coupling can lead to interesting $\mathbf{A B}$ oscillations of the linear conductance. If the electronelectron interactions are taken into consideration, further structure in the conductance may be expected due to the presence of an additional energy scale. Results in the regime in which such energy scale is relevant will be reported elsewhere.

\section{ACKNOWLEDGMENT}

This work was supported by a Cátedra Presidencial en Ciencias, FONDECYT 1990425 (Chile) and NSF grant No. 53112-0810 of Hunan Normal University (China). Discussions with P. Orellana are acknowledged. 


\section{REFERENCES}

[1] Y. Aharonov and D. Bohm, Phys. Rev. 115, 485 (1959).

[2] S. Washburn and R. A. Webb, Adv. Phys. 35, 375 (1986).

[3] C. W. J. Beenakker, H. van Houten, and A. A. M. Staring, Phys. Rev. B 44, 1657 (1991), and references therein.

[4] A. Yacoby, M. Heiblum, D. Mahalu, and H. Shtrikman, Phys. Rev. Lett.74, 4047 (1995);

R. Schuster, E. Buks, M. Heiblum, D. Mahalu, and H. Shtrikman, Nature 385, 417 (1997).

[5] G. Hackenbroich, Phys. Rep. 343, 463 (2001), and references therein.

[6] For a review, see L. P. Kouwenhoven, C. M. Marcus, P. L. McEuen, S. Tarucha, R. M. Westervelt, and N. S. Wingreen, in Mesoscopic Electronic Transport, Edited by L. L. Sohn, L. P. Kouwenhoven, and G. Schön, (Kluwer, Series E 345, 1997), P 105-214.

[7] D. V. Averin and Yu. V. Nazarov, Phys. Rev. Lett. 65, 2446 (1990).

[8] S. Datta, Electronic Transport in Mesoscopic Systems (Cambridge University Press, 1995), P246-273.

[9] L. P. Kouwenhoven, F. W. J. Hekking, B. J. van Wees, C. J. P. M. Harmans, C. E. Timmering, and C. T. Foxon, Phys. Rev. Lett. 65, 361 (1990); R. J. Haug, J. M. Hong and K. Y. Lee, Surf. Sci. 263, 415 (1992).

[10] C. A. Stafford and S. Das Sarma, Phys. Rev. Lett. 72, 3590 (1994); G. Klimeck, G. Chen, and S. Datta, Phys. Rev. B 50, 2316 (1994); F. R. Waugh, M. J. Berry, D. J. Mar, R. M. Westervelt, K. L. Kampman, and A. C. Gossard, Phy. Rev. Lett. 75, 707 (1995).

[11] T. H. Oosterkamp, T. Fujisawa, W. G. van der Wiel, K. Ishibashi, R. V. Hijman, S. Tarucha, and L. P. Kouwenhoven, Nature 395, 873 (1998); R. H. Blick, D. Pfannkuche, 
R. J. Haug, K.v. klitzing, and K. Eberl, Phys. Rev. Lett. 80, 4032 (1998); R. H. Blick, D. W. van der Weide, R. J. Haug, and K. Eberl, ibid. 81, 689 (1998).

[12] Z. Yu, T. Heinzel, and A. T. Johnson, Phys. Rev. B 55, 13697 (1997).

[13] Y. Meir and N. S. Wingreen, Phys. Rev. Lett. 68, 2512 (1992); A. -P. Jauho, N. S. Wingreen, and Y. Meir, Phys. Rev. B 50, 5528 (1994).

[14] Z. Y. Zeng, F. Claro, and W. Yan, e-print, cond-matt/0105194.

[15] D. N. Zubarev, Usp. Fiz. Nauk 71, 71 (1960) [Sov. Phys. Usp. 3, 320 (1960)].

[16] U. Fano, Phys. Rev. 124, 1866 (1961).

[17] (a) J. U. Nöckel and A. Douglas Stone, Phys. Rev. B 50, 17415 (1994); (b) J.Göres, D. Goldhaber-Gorden, S. Heemeyer, and M. A. Kastner, Phys. Rev. 62, 2188 (2000); (c) A. A. Clerk, X. Waintel, and P. W. Brouwer, Phys. Rev. Lett. 86, 4637 (2001).

[18] T. S. Kim and S. Hershfield, Phys. Rev. B 63, 245326 (2001); 


\section{FIGURES}

FIG. 1. Schematic plot of a $2 \times 2$ quantum dot structure enclosing a magnetic flux $\Phi$ with two terminals.

FIG. 2. Conductance $\mathcal{G}$ as a function of Fermi energy $\epsilon_{F}$ in the case of symmetric dot-lead couplings $\left(\Gamma_{L}=\Gamma_{R}\right)$ and in the absence of magnetic flux when the levels of the four dots are aligned (a) and not aligned (b, c).

FIG. 3. AB oscillations of the conductance $\mathcal{G}$ as a function of magnetic flux $\phi$ in the case of symmetric dot-lead couplings $\left(\Gamma_{L}=\Gamma_{R}\right)$. The structure parameters are the same as in Fig. 2 and the Fermi energy $\epsilon_{F}$ is set to be $0.1 \Gamma$.

FIG. 4. Evolution of the conductance $\mathcal{G}$ with the Fermi energy $\epsilon_{F}$ for the case of symmetric dot-lead couplings $\left(\Gamma_{L}=\Gamma_{R}\right)$ and the levels of the four dots aligned, and $\phi=0$ (solid), $\phi=\pi / 4$ (dashed), $\phi=3 \pi / 4$ (dotted) and $\phi=\pi$ (dash-dotted).

FIG. 5. Same as Fig. 4, but with the levels of the four dots not aligned.

FIG. 6. Conductance $\mathcal{G}$ in the cases of symmetric dot-lead couplings $\left(\Gamma_{L}=\Gamma_{R}\right.$, thin line) and asymmetric dot-lead couplings $\left(\Gamma_{R}=\Gamma_{L} / 2\right.$, thick line) for aligned four dot levels. The solid and dotted lines correspond to $\phi=\pi / 4$ and $3 \pi / 4$, respectively.

FIG. 7. Same as Fig. 6 but for non-aligned levels. 


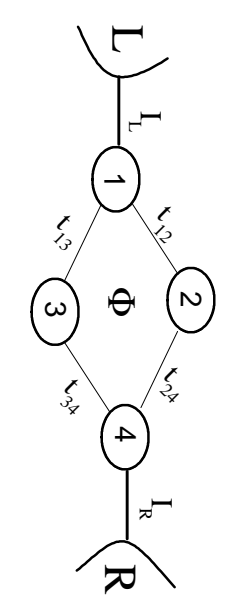

Fig. 1 Zeng et al. 
Fig. 2 Zeng et al.

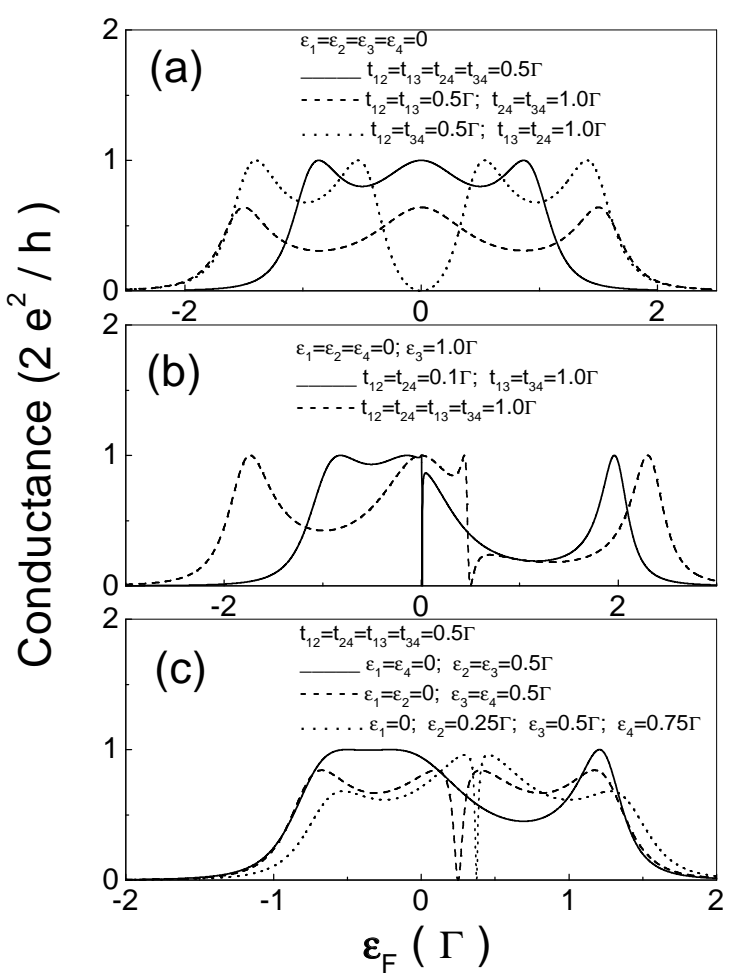


Fig. 3 Zeng et al.

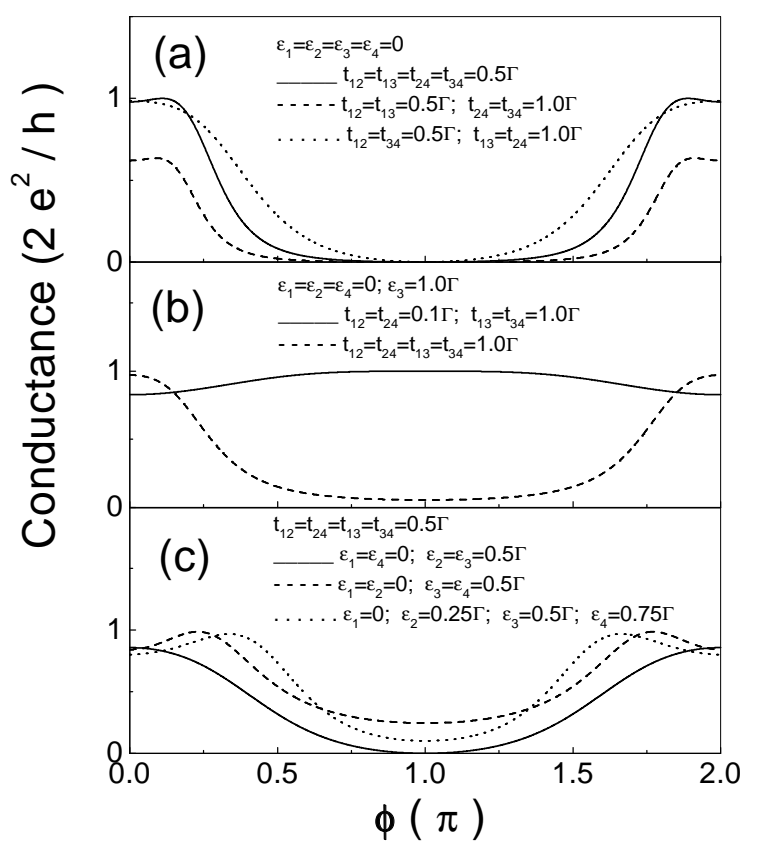


Fig. 4 Zeng et al.

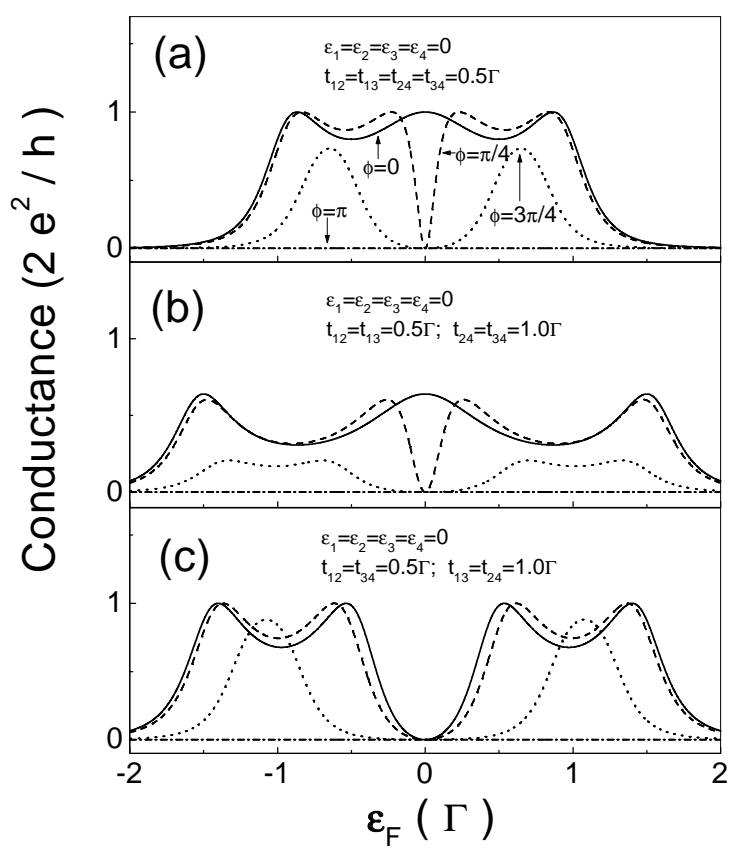


Fig. 5 Zeng et al.

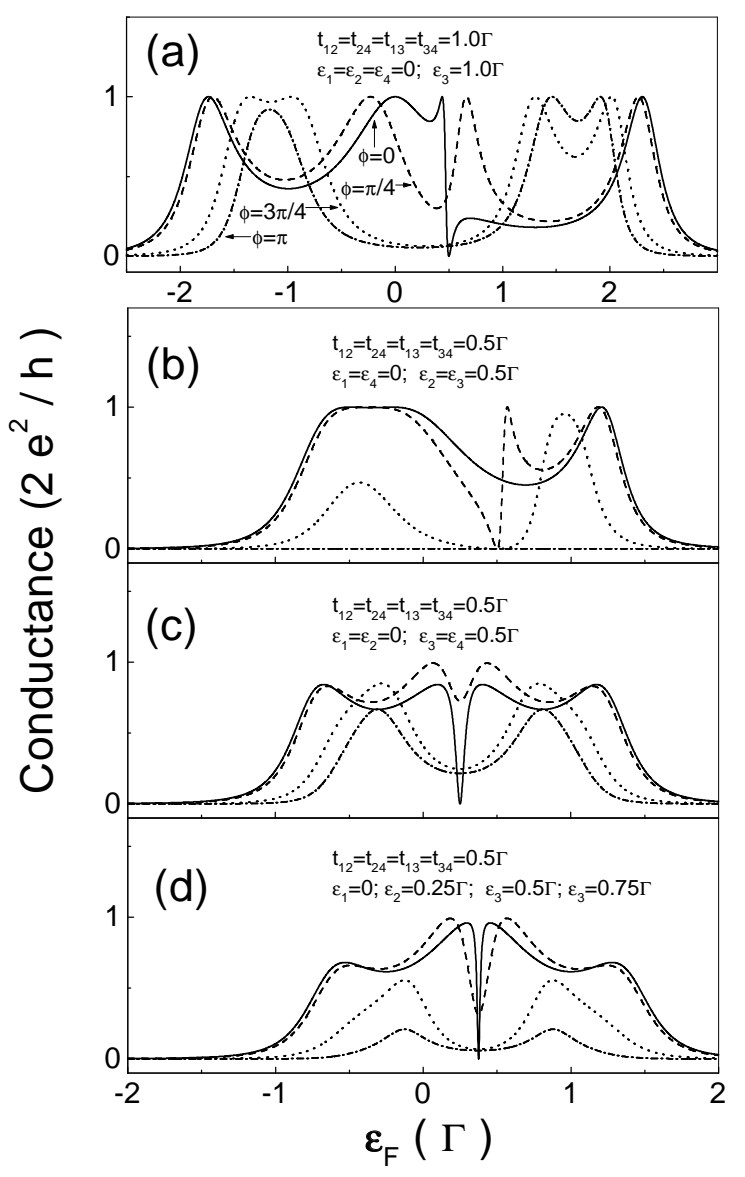


Fig.6 Zeng et al.

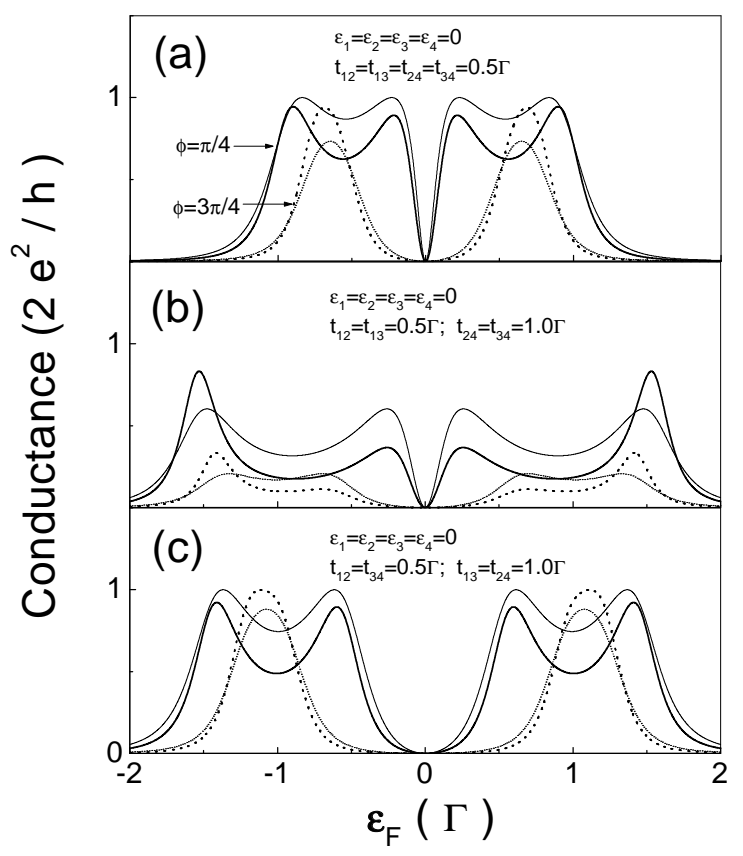


Fig. 7 Zeng et al.

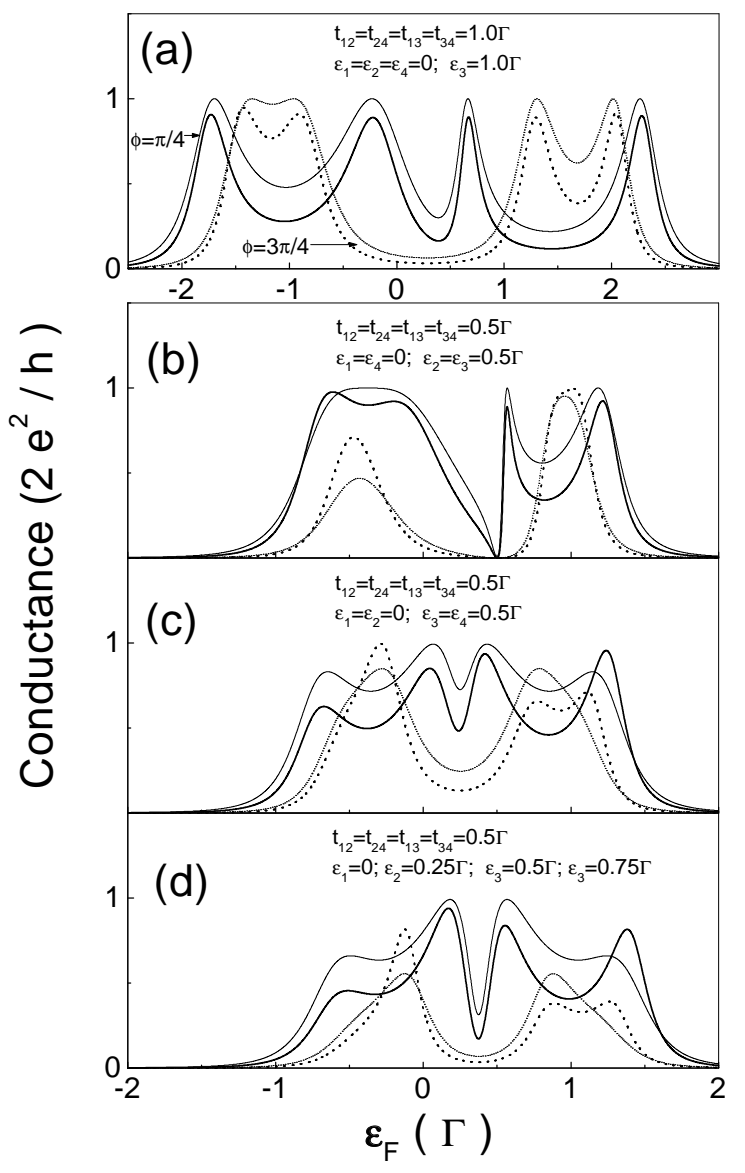

